\title{
Parques Industriales Ecoeficientes en Bogotá: ¿Una Alternativa Ambiental, Económica y/o Social?
}

\author{
Néstor Monroy*, Dayana Ramírez**
}

\begin{abstract}
Abstrac
El concepto de Parque Industrial Ecoeficiente (PIE), que en un principio se denominó ecoparque, ha evolucionado de este nombre ambientalista, a un enfoque más de ecoeficiencia; reconociendo que el tema ambiental no es la principal motivación de los empresarios y que requiere estar relacionado con la competitividad. Adicional a este reconocimiento, los PIE's de Bogotá tienen como base la respuesta a problemas sociales $y$ de planeamiento regional hacia una mejor calidad de vida y una mayor competitividad, no solo de las empresas, sino de la ciudad como tal. El análisis de las experiencias muestra que estos proyectos son a largo plazo, deben tener necesariamente un apoyo político, una cultura de participación y trabajo en equipo de las empresas y una organización centralizada. Igualmente se abre la posibilidad de PIE's o clusters "virtuales" los cuales no necesitan estar localizados en una misma área geográfica para cumplir sus funciones y objetivos.
\end{abstract}

\section{Palabras Claves}

PIE's, ecoeficiencia, competitividad, clusters industriales.

La actividad industrial ha sido fundamental en el desarrollo económico de los países, pero simultáneamente se ha convertido en uno de los mayores responsables del deterioro ambiental. Las emisiones al aire, las descargas a las fuentes hídricas, el uso del suelo, los niveles de ruido y la cantidad de residuos generados en la industria, han obligado a la humanidad a adoptar estrategias que aseguren el crecimiento económico sin poner en riesgo los recursos de las generaciones futuras, dando paso a la búsqueda del desarrollo sostenible.
Ante la problemática ambiental y el deseo de mejorar el desempeño económico de las industrias, surgieron los Parques Industriales Ecoeficientes $^{l}$ (PIE's), como una oportunidad para trabajar simultáneamente estos elementos. Hasta la fecha, aproximadamente 9 países han empezado proyectos de PIE's, todos con el objetivo de reactivar la economía de las regiones y proteger el medio ambiente.

\footnotetext{
Comunidad de empresas manufactureras y de servicios (localizadas en una misma área geográfica) *** buscando mejorar su desempeño económico y ambiental, a través de la colaboración en la gestión ambiental y temas de recursos incluyendo energía, agua y materiales... el grupo de negocios busca un beneficio colectivo que es mayor que la suma de los beneficios individuales que cada compañía alcanzaría si optimizará su desempeño individual

* Profesor Asociado Departamento de Ingeniería Industrial, Universidad de los Andes

** Ingeniera Industrial, Universidad de los Andes. 2003

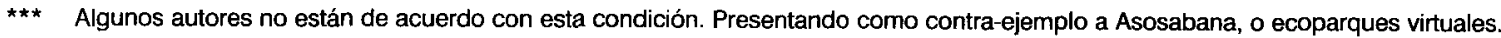


En el caso de Bogotá, los proyectos de Parques Industriales Ecoeficientes se han desarrollado como respuesta a diferentes problemáticas: el bajo desempeño ambiental de las industrias, especialmente micro, pequeñas y medianas empresas; la concentración de industrias en zonas residenciales, planes de renovación urbana y por políticas del Plan de Ordenamiento Territorial. Hasta el momento se han planteado 8 proyectos pero sólo Ascopro, inaugurado el 28 de Octubre, está en operación.

La implementación de un PIE, como cualquier otro proyecto, representa ventajas, desventajas y dificultades para los actores participantes. Sin embargo, el análisis de las experiencias y resultados obtenidos hasta el momento, permiten identificar factores claves de éxito y recomendaciones útiles para maximizar sus beneficios, $y$, minimizar los riesgos que representan su implementación y operación. Igualmente, se pueden hacer recomendaciones específicas para la Bogotá, que respondan a las condiciones específicas de la ciudad.

\section{Perspectiva Histórica}

El nacimiento de los PIE's ha surgido como respuesta a la necesidad de disminuir los impactos ambientales de los Parques Industriales $(\mathrm{PI})^{2}$, y a mejorar el desempeño ambiental de algunas industrias, que además de realizar procesos contaminantes, carecen de tecnologías apropiadas y están ubicadas inadecuadamente dentro de las ciudades.

Los primeros Parques Industriales aparecieron en el siglo XX, sin embargo su nacimiento se remonta a la baja edad media, cuando los artesanos empiezan a agruparse y se establecen reglamentos donde se reservaban el mercado local para los asociados, se evita la competencia y se fijan condiciones de trabajo. Con el transcurso de los años, los PI's empiezan a convertirse en el mejor instrumento para promover el desarrollo de la industria manufacturera, especialmente de las pequeñas empresas.

Actualmente, existen alrededor de $12.600 \mathrm{PI}$ 's en 90 países, caracterizados por estructuras y objetivos diferentes. Hoy en día, los PI's se caracterizan unos como zonas industriales, zonas francas, parques de negocios, parques de base tecnológica, zonas de exportación, parques de oficinas y clusters industriales, entre otros. Aunque en estas zonas se promueve el desarrollo económico, los impactos ambientales generados repercuten negativamente sobre la calidad del ambiente, obligando a varios parques a incluir el factor ambiental dentro de sus prioridades, convirtiéndose en PIE's. Otros PIE's han surgido por problemas de localización industrial, estancamiento económico, deterioro ambiental o reagrupamiento de empresas complementarias.

El primer proyecto piloto de PIE's se desarrolló en Kalundborg, Dinamarca, en el año 1970. Este PIE nació con el objetivo de mejorar el desempeño ambiental y económico de las empresas vinculadas. Sin embargo fue hasta 1993 cuando se formalizó el concepto de PIE por un equipo conformado por "Indigo Development", la Universidad de Dalhousie en Escocia y la Universidad de Cornell.

Hasta el momento, la mayoría de los PIE's desarrollados a nivel mundial se han caracterizado por la aplicación de los conceptos de ecología industrial y cero emisiones; mientras que en Colombia, la característica principal ha sido la reubicación y la aplicación de buenas prácticas ambientales.

Área geográfica delimitada que contiene varios negocios de naturaleza industrial, los cuales pueden ser diferentes o similares, livianos o pesados, modernos o relativamente obsoletos; pero el elemento esencial es que el parque se administra por parte de una sola aubridad que tiene definida su jurisdicción con respecto a las compañías residentes. 


\section{PAROUES INDUSTRALES ECOEFICIENTES EN EL Mundo}

Varios países han adelantado proyectos bajo la concepción de PIE's. Para 1996, 17 proyectos se declararon como PIE's en Estados Unidos, y a finales del 2001 en Asia, Europa, Estados Unidos, África y Sur América ya se habían iniciado proyectos y otros programas y planes para el desarrollo eco-industrial (DAMA).

Hasta Junio de 2002, los PIE's identificados eran aproximadamente 51 , cada uno con elementos distintivos, que permitió su agrupación en 9 clases. Ver Tabla 1. Es posible que un mismo parque tenga características de diferentes modelos, y teniendo en cuenta la evolución que han seguido los PIE's, es posible que los proyectos del futuro, se caractericen por reunir varias o todas las características de los que actualmente han sido identificados.

Tabla 1. Tipos de PIE's

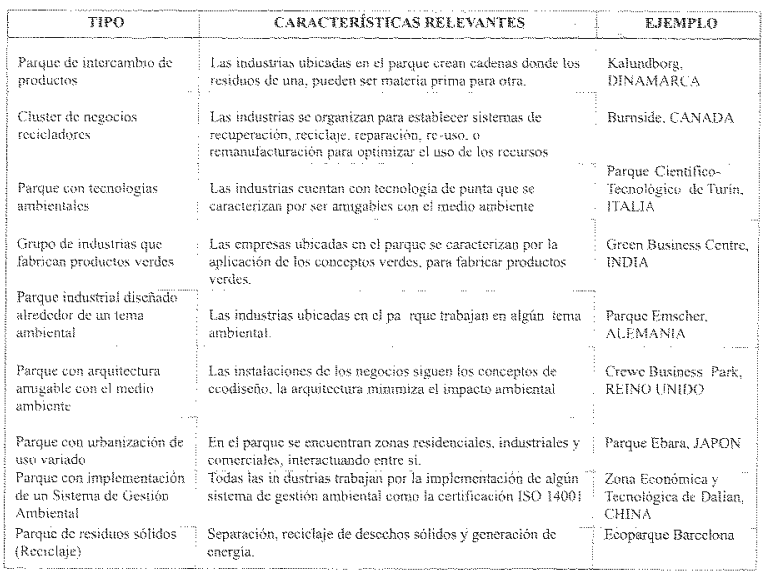

También existen otros PIE's virtuales, caracterizados porque las empresas no están ubicadas geográficamente en la misma zona, pero están organizadas de tal forma, que pueden establecer sinergias entre ellas.

Los resultados obtenidos con la implementación de PIE's han sido diversos: ahorro en recursos y materias primas, eficiencia en los procesos, relaciones con la comunidad, capacitación de empleados, formalización de empresas, reconocimiento por desempeño ambien- tal, aumento de inversión y posicionamiento de las empresas. En el Anexo 1, se describen brevemente los proyectos y los resultados obtenidos en cada uno.

\section{PAROUES INDUSTRIALES ECOEFICIENTES EN Gogora}

En Bogotá los PIE's han nacido en su mayoría por la necesidad de reubicar las empresas siguiendo las políticas establecidas en el Plan de ordenamiento Territorial y, por problemas con las comunidades vecinas. La reubicación se ha convertido en una oportunidad para mejorar el desempeño ambiental de las empresas; su traslado y operación, puede caracterizarse por la creación de sinergias y la aplicación de prácticas amigables con el ambiente. El trabajo en conjunto de las empresas, mejora su desempeno ambiental y disminuye costos de producción, mejorando así, su rentabilidad e imagen.

Los proyectos planteados en la ciudad de Bogotá, se pueden dividir de acuerdo a la agrupación por sectores: multisectoriales o unisectoriales; y, según su origen: reglamentación del POT o reubicación de empresas. Ver gráfica 1 .

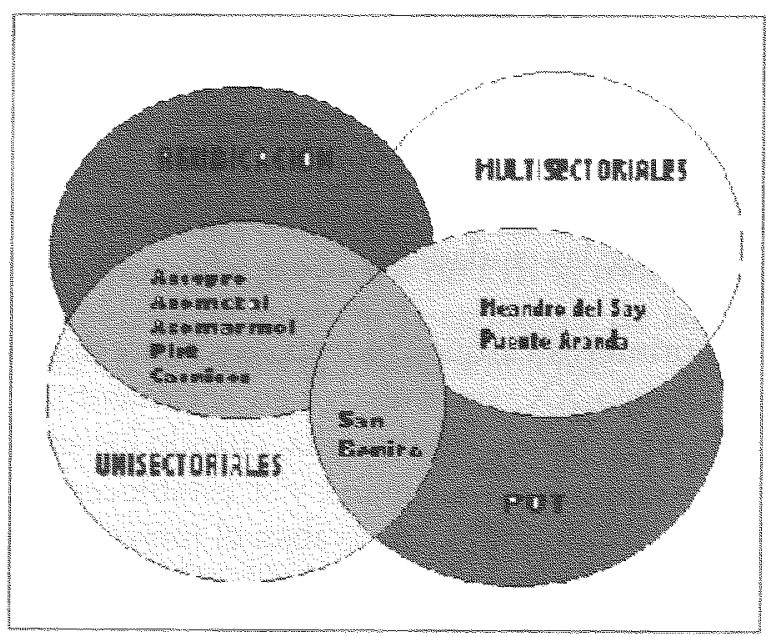

Gráhea 1. Características De Los PIE'S en Bogotá

En la tabla del Anexo 2 se resume los resultados obtenidos en los proyectos planteados para Bogotá. 
En la ciudad capital, el desarrollo de PIE's se ha consolidado con el programa Parques Industriales Ecoeficientes para Bogotá, el cual fue planteado a partir del Plan de Ordenamiento Territorial de Bogotá, el Plan de Gestión Ambiental del Distrito 2001-2009 y la Política de Producción y Consumo Sostenible para Bogotá. Estos se convierten en una estrategia para fortalecer el desarrollo económico de la ciudad asegurando la calidad ambiental y la calidad de vida de los ciudadanos. De igual forma, se espera que el programa continúe orientando hacia la transformación de la ciudad, como un ecosistema urbano sostenible, bajo el liderazgo del DAMA.

\section{Ventajas, Desventajas y Dificultades DeL PIE}

De acuerdo al análisis de los diferentes sectores, las condiciones de los empresarios y la caracterización de los factores críticos de éxito, se pueden identificar varias ventajas en la construcción de un PIE. Este, además de ser una oportunidad para fortalecer el sector, es una alternativa para la solución de los problemas de localización industrial y una opción para controlar los impactos ambientales generados por la actividad industrial.

A pesar de las ventajas de la consolidación de un PIE, este también representa algunas dificultades. La construcción del PIE debe llevarse a cabo en lugares destinados para la actividad industrial, en Bogotá, esto representa una gran dificultad por el costo y disponibilidad de zonas adecuadas. Además, la construcción de un PIE, demanda un aporte de capital por parte de los empresarios, quienes a pesar de reconocer sus beneficios, carecen de recursos económicos, o tienen necesidades más urgentes.

A continuación se presentan las ventajas y dificultades más significativas en la construcción de un PIE en la ciudad de Bogotá.

\section{Ventajas}

Como se menciona anteriormente, la consolidación de un PIE brinda grandes ventajas a los empresarios, autoridades y comunidad, entre los beneficios más significativos encontramos:

\section{Gestión empresarial}

- La organización de las empresas les permite aumentar su poder de negociación con los proveedores.

- La estructura del PIE, agrupa a las empresas de tal manera que forman una sola empresa de gran envergadura. Esto les puede facilitar el acceso a fuentes de financiación para incentivar el desarrollo individual y del PIE.

- La unión de los esfuerzos de cada empresa, puede favorecer la conformación de grupos de capacitación, seguridad industrial y primeros auxilios con el fin de enfrentar cualquier situación riesgosa, con eficacia.

- El PIE incentiva la formalización de las empresas, mejorando su imagen. Esto puede influir directamente en el aumento de clientes, que ven en el parque una alternativa confiable para acceder a productos $y$ servicios de calidad.

- La conformación del PIE, puede atraer inversión extranjera y promover el apoyo de organizaciones relacionadas con la innovación tecnológica, mejorando los procesos desarrollados por las empresas.

- La organización de los empresarios puede servir para presionar por la obtención de beneficios tributarios, disminución de impuestos e incentivos económicos.

- La consolidación del PIE involucra la creación de un centro de asesoría que sería una herramienta fundamental para apoyar y asegurar el buen funcionamiento de las empresas. De igual forma, puede mantener a las empresas en continua actualización sobre nuevas tecnologías y procesos.

- Se puede establecer una red de información que le permita a los empresarios tener acceso continuo a proyectos, actualizaciones y estadísticas, que les permita tomar 
decisiones adecuadas para asegurar el desarrollo de sus empresas.

Gestión ambiental

- La organización de empresas permite implementar programas para minimización de recursos y manejo de residuos con mayor fa- cilidad que para las empresas individuales.

- La creación de una planta de tratamiento de aguas y una planta de energía mejora el uso de recursos naturales y evita que se continué deteriorando el ambiente, asegurando en cierta medida la disponibilidad de recursos para las generaciones futuras.

Cuadro 2. Áreas Potenciales para mejorar el desempeño empresarial

MATERIALES

Compras Comunes

Relaciones proveedor/cliente

Conexiones subproductos

Creación mercados de materiales nuevos

TRANSPORTE

Transporte compartido

Mantenimiento compartido

Alternativas de empaque

Transporte dentro del Parque

Logística integrada

Despachos conjuntos

ENERGÍA

Construcciones verdes

Ahorro de energía

Cogeneración

Combustibles alternativos

Seguimiento
CALIDAD DE VIDA RELACIONES CON LA COMUNIDAD

Integración trabajo y recreación

Oportunidades de educación cooperativa

Programas con la comunidad

Planeación regional con la comunidad

RECURSOS HUMANOS

Reclutamiento

Benéficos conjuntos

Programas de salud

Empleo

Seguridad, bienestar

Capacitación

\section{MARKETING}

Sellos verdes

Acceso a mercados verdes

Promociones

Riesgo compartido

Reunir empresas que agreguen valor

\section{PROCESOS DE PRODUCCIÓN}

Prevención de contaminación

Reducción de desechos y re-uso

Diseños de procesos

Subcontratación conjunta

Equipos

Transferencia e integración de tecnología

AMBIENTE / SALUD / PROTECCIÓN

Construcciones verdes

Ahorro de energía

Cogeneración

Combustibles alternativos

Seguimiento 
- La construcción del Parque puede involucrar desde su inicio, el uso de materiales amigables con el ambiente, la minimización de recursos, y además caracterizarse por una arquitectura verde, sirviendo de ejemplo para la construcción de nuevas infraestructuras.

- La concentración de varias industrias en el mismo lugar, puede facilitar la acción de las autoridades en materia de control y seguimiento y a su vez demandar menos recursos para tales acciones.

Gestión social

- La agrupación de los industriales en un PIE, fortalece las medidas de seguridad que además de proteger a los empresarios y el PIE, favorecen la seguridad del sector.

- El PIE puede presionar para mejorar la prestación de servicios públicos y el estado de vías de acceso. EL número de empresas favorece la solicitud para mejorar la red telefónica, la implantación de una planta de energía y la construcción de nuevas vías o pavimentación de las existentes.

- Los beneficios económicos generados al pertenecer al PIE, pueden mejorar la situación actual de las industrias evitando que muchas de ellas cierren, de tal forma que evita el crecimiento del desempleo.

Gestión regional

- La organización de las empresas favorece su capacidad de respuesta frente a políticas reglamentarias relacionadas con el área económica y ambiental, y tendencias del mercado.

- El fortalecimiento de las pequeñas industrias ayuda con la reactivación económica de las regiones, en cuanto genera empleo, promueve la tecnificación, brinda seguridad a los trabajadores, colabora con el desarrollo de las comunidades.

En el cuadro 2, se resumen las áreas potenciales donde un PIE puede crear relaciones para mejorar el desempeño individual de las empresas y en conjunto del Parque.

\section{Desventajas}

Aunque los beneficios son considerables, y las ventajas favorecen indiscutiblemente el desarrollo económico de los sectores que están involucrados en el parque, y por ende el desarrollo de la ciudad, su implementación en la ciudad de Bogotá, debe enfrentar como desventaja principal que la concentración de empresas en un mismo lugar puede aumentar la presión local sobre los recursos naturales, lo que demanda mayor atención y esfuerzo de los empresarios y de las autoridades.

\section{Dificultades}

De igual forma, la implementación y operación de un PIE debe enfrentar algunas dificultades que si bien no son determinantes, si han obstaculizado los proyectos desarrollados hasta el momento en la ciudad capital:

- El costo de las zonas industriales.

- La mayoría de las empresas son micro, pequeñas y medianas empresas que carecen de capital de para invertir en proyectos de PIE's.

- La ubicación de empresas que realizan actividades similares en el mismo lugar, puede agudizar la competencia.

- La mentalidad tradicional de los empresarios, caracterizada por la importancia de los beneficios económicos dificulta la visión donde la problemática ambiental influye directamente sobre la rentabilidad de las industrias.

- Baja presión para que los empresarios actualicen las tecnologías.

- La creación de relaciones entre las empresas puede promover la dependencia entre ellas.

- Los beneficios ambientales del PIE pueden ser muy buenos, pero su desarrollo económico insostenible.

- La dependencia de entidades públicas y poco liderazgo de las empresas privadas puede afectar el sentido de apropiación del PIE y la continuidad de las políticas. 
- Las decisiones sobre el PIE deben agrupar estrategias no solo en materia ambiental sino en diferentes campos y niveles para lograr el desarrollo sostenible del mismo.

- La adquisición de tecnologías avanzadas demanda personal capacitado para su mantenimiento, esto puede representar un costo adicional para el funcionamiento del PIE.

- Mentalidad generalizada de los empresarios de aplicar soluciones de fin de tubo para manejar impactos ambientales.

- Falla en la coordinación con otras entidades Distritales, especialmente en lo relacionado con la reglamentación de las Unidades de Planeación Zonales (UPZ).

- Escasa difusión del concepto y del proyecto a nivel Distrital, tanto en el sector público como privado.

\section{Experiencias Obtenidas}

Del cuadro de resultados de los PIE's en Bogotá, mostrado en el anexo 2 , y de las reuniones y foros con las diferentes partes interesadas (empresas, DAMA, comunidades y académicos) se pueden sintetizar las siguientes lecciones:

1. Delimitado en su alcance y objetivos.

2. Es un proceso continuo a largo plazo

3. Requiere trabajo conjunto: publico - privado (no es normativo, es concertado)

4. Debe incluir un centro de servicios y capacitación

5. Manejo Integral (ambiental, calidad, salud ocupacional, seguridad industrial, movilidad)

6. Requiere un encadenamiento industrial

7. No es necesaria una reubicación de las empresas, pero es un excelente motivador.

8. Administración centralizada

9. Infraestructura compartida

10. Es indispensable aplicar herramientas de ecoeficiencia

11. Desarrollo y medición de Indicadores.

Adicionalmente, se han identificado los siguientes beneficios para la Ciudad: Ordenamiento, Competitividad de la ciudad, Calidad de vida, Reconocimiento internacional.

\section{Recomendaciones}

Después de la documentación de los casos desarrollados a nivel mundial y en Bogotá, y de la consulta de diferentes documentos relacionados con PIE's, se desarrollaron algunas recomendaciones útiles para la implementación y operación de estos. Dichas recomendaciones, si bien no pueden garantizar el éxito absoluto del PIE, donde inevitablemente intervienen factores indeterminados y difíciles de manejar, si pueden ser útiles para convertir las desventajas y dificultades en oportunidades, y aprovechar las ventajas para optimizar el funcionamiento de las compañías, con el objetivo de convertir el PIE en un modelo de desarrollo sostenible.

Según Cote, existen 3 elementos indispensables para establecer un PIE:

1. Manejo de Información: Es necesario conocer el flujo de materiales, los productos nuevos y usados como también el exceso de los mismos, para lograr que las empresas establezcan relaciones efectivas entre ellas y con la comunidad.

2. Instrumentos económicos: Deben lograr que el poder del mercado presione a las empresas para desarrollar actividades amigables con el ambiente.

3. Regulaciones: Las políticas ambientales deben estar orientadas hacia la prevención y reducción de la contaminación más que hacia su tratamiento.

De igual forma, Cote propone otras estrategias para la implementación de un PIE, útiles para convertirlos en un modelo de desarrollo sostenible:

- Ubicación: El lugar debería ser seleccionado teniendo en cuenta las características topográficas y disponibilidad de recursos naturales.

- Diseño: Debería contener características naturales que asimilen el PIE con un ecosistema. 
- Construcción: Debe minimizar el uso de materiales y desarrollarse aplicando conceptos ecológicos.

- Conservación: Los sistemas de producción y transporte deben diseñarse para minimizar energía y recursos.

- Sustitución / uso de productos ambientales: Los insumos más contaminantes deben ser sustituidos por otros menos tóxicos y más degradables.

- Auditorias: Es conveniente establecer un sistema de control que garantice la eficiencia de los procesos y uso de materiales.

- Estandarización: Estandarizar materiales y procesos para reducir desperdicios y facilitar el intercambio.

- Control de Inventarios: Reducir la cantidad de materiales dentro del parque disminuye el riesgo de accidentes y evita la degradación de los mismos.

- Intercambio de materiales: las empresas deberían usar los residuos generados dentro del parque, estableciendo relaciones que les permita intercambiar o transferir materiales.

- Colección de información: El buen funcionamiento de un PIE depende en gran parte del conocimiento sobre materiales usados, energía requerida y residuos generados.

- Co-localización de negocios: Si es posible, los negocios debería ubicarse en el parque de tal forma que puedan maximizar el uso de calor y aguas residuales.

- Promover la compra, venta y comercio de desperdicios, al igual que la reparación y mantenimiento de materiales.

- Retroalimentación y comunicación: Es importante que las empresas tengan buena comunicación entre ellas, con el parque y la comunidad. De esta forma, se pueden aprovechar mejor las oportunidades y responder con efectividad a las necesidades de la comunidad, donde pueden estar los clientes potenciales del PIE.

El éxito de un PIE no es asegurado por la aplicación de principios generales. Lo que para un parque puede ser una ventaja, para otro puede representar un obstáculo para su óptimo desa- rrollo. Sin embargo, la presencia de algunos elementos puede facilitar el trabajo cooperativo y la generación de beneficios para las partes involucradas. De acuerdo a los casos profundizados, son factores claves de éxito:

- Trabajo en Equipo entre las industrias, el gobierno, la academia y la comunidad: El trabajo conjunto de estos elementos garantiza que los intereses de las 4 partes sean maximizados sin perjudicar a las demás, de esta forma se obtiene lo que hoy en día es imprescindible para garantizar las generaciones futuras: el desarrollo sostenible.

- Tecnología avanzada: Para lograr que un PIE trabaje como un ecosistema natural, se necesita trabajar con tecnologías de punta que permitan imitar las relaciones complejas, características de la ecología natural.

- Mente abierta y disposición por parte de las industrias participantes: Las empresas deben tener una motivación individual para participar en el PIE y estar dispuestas a crear relaciones de cooperación que les permita maximizar sus beneficios.

De acuerdo a lo anterior, a las experiencias a nivel mundial y los resultados obtenidos, (Anexo 1) y, el contexto económico, social y ambiental de la ciudad capital, se identificó un conjunto de recomendaciones para la ciudad de Bogotá, que podrían mejorar el proceso que hasta el momento han venido siguiendo los PIE's:

1. Aprovechar las condiciones naturales de la ciudad: En el PIE, Meandro del Say, debería incorporar como elemento característico el paisaje del humedal y fomentar su protección, como se hizo en el caso del PIE Crewe Business Park, en el Reino Unido.

2. Sensibilización sobre beneficios económicos: Todas las empresas trabajan por tres objetivos principales: rentabilidad, crecimiento y sostenibilidad, por lo que siempre estará primero el factor económico sobre el ambiental. Esto significa, que para animar a los empresarios a vincularse en 
proyectos de PIE, se debe mostrar primero los beneficios económicos, y posteriormente los ambientales.

3. Fortalecer líneas de crédito: La mayoría de empresas vinculadas en proyectos de PIE's son pequeñas y medianas empresas, que no cuentan con capital para invertir. Teniendo en cuenta que la construcción de un PIE, demanda una inversión alta (entre $\$ 4.000$ y $\$ 12.000$ millones de pesos) y requiere un aporte inicial por parte de cada negocio participante, es necesario ofrecerles líneas de crédito que les permita incorporarse en el proyecto. Un ejemplo es el caso de ASCOPRO, donde el 53\% fue financiado con la línea de crédito IFI-DAMA.

4. Crear políticas que incentiven a los empresarios y disminuyan el costo de los terrenos: Una de las principales dificultades para iniciar proyectos de PIE en Bogotá, es el costo de los terrenos (entre $\$ 1500$ y $\$ 2000$ millones de pesos). Para solucionar esto, se debería crear una bolsa de terrenos, donde los costos sean asequibles para los empresarios y brinden diferentes alternativas para establecer el PIE.

5. Buscar apoyo en las Universidades: Las universidades pueden trabajar en el desarrollo de nuevos procesos y en la optimización de los existentes, en la innovación de tecnologías, en el estudio de nuevas sinergias entre empresas y en la caracterización de los residuos, como se hizo en el caso internacional de Burnside, Canadá. Además, pueden colaborar en la capacitación de los trabajadores y empresarios del PIE

6. Trabajo en equipo entre Gobierno, Universidad, empresarios y comunidad: El buen funcionamiento de un PIE, requiere que todos los actores maximicen sus beneficios7., de tal forma que las decisiones que se tomen no perjudiquen a ningún actor, evitando que se repita lo ocurrido con el lote adquirido por ASOMETAL.
8. Continuidad de políticas, a pesar de los cambios de gobierno: Para lograr que los empresarios participen activamente en el PIE, es necesario asegurarles que el proyecto tendrá continuidad en el tiempo.

9. Reconocimiento a los empresarios: Es muy importante motivar a los empresarios, no sólo con respecto a los beneficios económicos y ambientales, sino además con el mejoramiento de la imagen empresarial, el reconocimiento por su desempeño a través de premios y certificaciones, y preferencias para negociaciones con el gobierno.

\section{Conclusiones}

La implementación de un PIE genera amplios beneficios económicos, ambientales y sociales, pero también representa dificultades que demandan el trabajo continuo de las industrias, el gobierno y la comunidad. Solo la concientización sobre la importancia del desarrollo sostenible y la identificación del factor ambiental como una oportunidad para activar la economía de la ciudad, pueden asegurar que el PIE se convierta en una herramienta eficaz para mejorar la competitividad de las industrias. Debido a la resistencia al cambio de los industriales, es imprescindible mostrar primero las bondades económicas que genera la implementación del PIE y posteriormente los beneficios ambientales, los cuales finalmente se traducen en ahorro en costos y aumento de rentabilidad para las industrias.

Los PIE's también representan una excelente oportunidad para mejorar el manejo de los factores competitivos de la ciudad. La capacitación de trabajadores y empresarios, fortalece el conocimiento y se convierte en terreno fértil para la innovación. La construcción de un parque con arquitectura verde puede representar un lugar turístico y cultural que atraiga mayor número de visitantes, el mejoramiento de los servicios públicos y la malla vial facilitan la conexión y desarrollo de operaciones dentro de la ciudad, la inversión en el PIE fortalece el 
posicionamiento de las empresas y apoya su internalización; y el uso eficiente de recursos y manejo de residuos, aseguran mejor calidad del ambiente, atrayendo así, inversionistas extranjeros.

Hasta el momento se han identificado 9 tipos de PIE's, los cuales a pesar de sus diferencias, han logrado mostrar que la característica principal de estos, es la integración del factor ambiental dentro de las estrategias empresariales para obtener beneficios económicos sociales y ambientales, convirtiéndose en modelo de desarrollo sostenible. Aunque todos los PIE's tienen un elemento distintivo, varios de ellos reúnen características de diferentes tipos, demostrando que los PIE's están en continua evolución y constante búsqueda de oportunidades. Todos procuran mejorar su desempeño económico y ambiental, de acuerdo al contexto particular en el que se desenvuelven.

Los PIE's representan una buena estrategia para mejorar el desempeño ambiental de las empresas y promover el desarrollo económico de las regiones. Aunque el desarrollo de PIE's está en su etapa temprana, es indiscutible la importancia que ha venido tomando en lo últimos años. De igual forma, la evolución del concepto, promete el desarrollo de nuevos proyectos con características innovadoras, como lo están siendo los PIE's virtuales, donde la ubicación geográfica en el mismo lugar no es un factor imprescindible, por el contrario, representa una oportunidad para que más empresas puedan vincularse al Parque a través de su participación en el flujo de recursos.

\section{Bibliografia}

- Néstor Monroy y Dayana Ramírez. Universidad de los Andes. 2003. Documentación y análisis de Parques Industriales Ecoeficientes a nivel mundial y en Bogotá

- DAMA, Seminario PIE’s para Bogotá. 2004

- DAMA, Documento PIE's . 2003

- R. Cotte. Ecoindustrial Strategies. Greenleaf Publishing.
ANEXO l:

\section{RESULTADOS CASOS INTERNACIONALES}

\section{KALUNDBORG, DINAMARCA}

Consiste en el intercambio de productos y cooperación entre las 4 empresas participantes, Asnaes, compañía de generación eléctrica; Novo Nordisk, planta farmacéutica; Gyproc planta que produce tableros y la refinería Statoi.

Algunos resultados:

- Reducción de demanda de agua entre un 20 y $25 \%$ de las empresas participantes

- Reducción de consumo de aceite en 19000 toneladas por año

- Reducción consumo de carbón en un 2\%

- Reducción de emisiones al aire de CO2 en 3\%

- Reducción de emisiones al aire de SO2 en $58 \%$

- Conversión de desperdicios a materia prima: 135000 toneladas de cenizas por año, 2800 toneladas de sulfuro por año, 800 toneladas de nitrógeno y 400 toneladas de fósforo sirven como fertilizantes

- Trabajo cooperativo entre las empresas para brindar seguridad y capacitación a los empleados.

\section{BURNSIDE, CANADÁ}

Busca la creación de relaciones entre las empresas y el medio ambiente, para convertir el parque en un ecosistema industrial. Promover el reciclaje, el re-uso, recuperación, reparación, re-manufacturación, renta.

Resultados:

- Creación del Centro de Eco-eficiencia

- Publicación de revista sobre temas ambientales y ecoeficiencia.

- Intercambio de productos, especialmente materiales de empaque (cartón, envases de plástico y metal, filtros)

- Empresas de muebles redujeron las aguas residuales con pegamento en $83 \%$, y disminuyeron uso de solventes químicos.

- Materiales metálicos son vendido a una empresa de salvamento para reparación y venta.

- Los envases son reciclados eliminando los costos asociados con su envió a rellenos sanitarios.

- $\quad 15 \%$ de las empresas ofrecen servicios de reciclaje, el re-uso, recuperación, reparación, re-manufacturación.

- Varias zonas del parque han sido restauradas para ofrecer oportunidades de recreación a trabajadores y visitantes.

\section{PARQUE AMBIENTAL TURÍN ITALIA}

Pretende integrar el factor ambiental a los procesos de producción y prestación de servicios a través de la investigación y transferencia de tecnologías a las pequeñas y medianas empresas.

Proyectos adelantados:

- Construcción del parque aplicando los conceptos de ecodiseño. 
- Investigación en el re-uso del asbesto.

- Uso de sensores a remoto para aplicaciones ambientales.

- Diseño en un curso de capacitación en temas ambientales.

- Investigación en el control de la contaminación del aire, usando bio-detectores.

- Uso de biomasa vegetal para la generación de energía usando una planta gasificadota.

- Evaluación de la presencia de sustancias metálicas en el aire usando musgo como biodetector.

\section{PARQUE ECO-INDUSTRIAL EBARA, JAPÓN}

Implementación del programa ZERI en los procesos productivos de la corporación, convirtiendo 35 hectáreas de su propiedad en un PIE. Construcción del PIE está proyectada para el año 2010, y busca incluir viviendas, industrias, agricultura, servicios públicos, deportes y recreación, y, áreas naturales.

Resultados esperados:

- Reducir el consumo de energía en $40 \%$

- Reducir consumo de agua en 30\%

- Reducir descarga de residuos en 95\%

- Reducir emisiones de dióxido de carbono en $30 \%$

\section{ZONA DE DESARROLLO ECONÓMICO Y SOCIAL} DE DALIAN

Implementación sistema de gestión ambiental, ISO 14001 Standard.

Resultados:

- Inversión US \$24.18 millones en la construcción de 2 plantas de tratamiento

- $\quad 1.2$ millones de toneladas de agua reciclada cada año

- Aumento 98\% uso de gas domestico

- 13 empresas certificadas

- Implementación del programa Concientización y Preparación para Emergencias a Nivel Local.

- Forestación de $4662 \mathrm{~km} 2$

- Disminución $46.5 \%$ partículas suspendidas, $16.7 \%$ de dióxido de sulfuro, $13.2 \%$ de CO

- Aumentó de inversión, por ejemplo Kaijin Chemical invirtió 500 millones de RMB para expandir sus negocios, Chrysler Motor Corp. Invirtió US \$150 millones.

\section{PARQUE EMSCHER, ALEMANIA}

Reestructuración de la zona de Emscher, por medio del desarrollo de proyectos ecológicos y de regeneración económica. En 1993, 134 proyectos se habían desarrollado con la inversión de DM 2.5 billones, de los cuales DM 1.7 millones provenían de fuentes públicas.

Proyectos adelantados:

- Landscape Emscher Park: Transformación de espacios abiertos en zonas recreativas

- Museo Industrial y Preservación histórica: Las industrias se transformaron en museos industriales y centros de actividad artística
- Prosper III, Brownfields : incluir una zona de vivienda dentro del parque

- Puerto de Duisborg: revitalizar la zona ribereña de Duisborg

- Desarrollo ecológico de ríos navegables de Emscher: reingeniería ecológica del sistema de aguas de Emscher.

\section{CENTRO DE NEGOCIOS VERDE}

Aplicación del concepto verde en las actividades de las empresas, y en la construcción del centro de negocios.

Algunos resultados:

- cambios dentro del sistema que permitan un ahorro de energía entre 5 y $15 \%$

- Adopción de tecnología avanzada, obteniendo un ahorro de 30 a $40 \%$ en energía.

- Uso de tecnologías de generación de energía renovable como molinos (potencial de la India $20000 \mathrm{MW}$, de los cuales solo se han usado $600 \mathrm{MW}$ ), energía solar( potencial de India 5000 trillones de unidad por año), y, biomasa (potencial de India $16000 \mathrm{MW}$, instalado $222 \mathrm{MW}$ )

- Tratamiento de agua

- Reciclaje.

\section{CREWE BUSINESS PARK}

Posee Arquitectura amigable con el medio ambiente, los negocios deben desarrollar infraestructura que sea amigable con el ambiente y su diseño debe tener en cuenta las características naturales del parque, como el arroyo del Valle, los árboles de roble y los prados.

Resultados:

- Al menos 22 clases de mariposas y 16 variedades de libélulas se han registrado.

- 40 especies de pájaros se albergan en el parque

- Se han visto especies poco conocidas como Wheatear, Waxwing, Whinchat, y Redstart.

- Excelente desempeño ambiental, obteniendo el premio nacional Pondwatch, el premio Bloom NorthWest Committee's New Landscaping y el premio Millenium Marque.

\section{BARCELONA}

Instalaciones concesionadas por 15 años para el manejo de los residuos municipales. Con una inversión de 62 millones de dólares, cuenta con 3 líneas principales: Reciclaje, mecanización - fermentación y compostaje.

Sus principales objetivos son: ser flexible a diferentes tipos de residuos, maximizar el Biogas y minimizar los desechos orgánicos. '

Algunas características importantes:

- Cuenta con una integración arquitectónica

- Sus ingresos provienen de: costo de tonelada tratada, la venta del reciclaje y la producción de energía 
ANEXO 2.

RESULTADOS DE PIE'S EN BOGOTÁ

\begin{tabular}{|c|c|c|c|}
\hline PROYECTO & $\begin{array}{c}\text { ASCOPRO } \\
\text { (2001) }\end{array}$ & $\begin{array}{c}\text { SAN BENITO } \\
(\mathbf{2 0 0 0 )}\end{array}$ & $\begin{array}{c}\text { ASOMETAL } \\
\text { (2000) }\end{array}$ \\
\hline ORIGEN & $\begin{array}{l}\text { Reubicación de los empresarios } \\
\text { de artes gráficas por la } \\
\text { construcción del Parque Tercer } \\
\text { Milenio. }\end{array}$ & $\begin{array}{l}\text { Designación de la zona para ejecutar } \\
\text { programa de Ecoparques. } \\
\text { Reglamentado por el Plan de } \\
\text { Ordenamiento Territorial (POT) }\end{array}$ & $\begin{array}{l}\text { 1993: proyecto para construir } \\
\text { ciudadela Industrial. } \\
\text { 2000: Acercar decidió continuar } \\
\text { proyecto de Ciudadela pero } \\
\text { enfocándolo hacia un PIE. }\end{array}$ \\
\hline OBJETIVOS & $\begin{array}{l}\text { - Arquitectura verde } \\
\text { - Producción más limpia } \\
\text { - Programa de buenas prácticas } \\
\text { ambientales, sustitución y } \\
\text { reducción de insumos y } \\
\text { reutilización y reciclaje de } \\
\text { residuos. } \\
\text { - Encadenamiento productivo } \\
\text { - Centro Tecnológico de Artes } \\
\text { Gráficas. } \\
\text { - Implantación de sistemas de } \\
\text { control de residuos líquidos } \\
\text { - Diseño de una red eléctrica } \\
\text { para minimizar el consumo de } \\
\text { energía. }\end{array}$ & $\begin{array}{l}\text { - Implementación de un programa de } \\
\text { minimización de residuos } \\
\text { - Desarrollo de una propuesta de } \\
\text { innovación tecnológica } \\
\text { - Implementación de un centro de } \\
\text { investigación y desarrollo } \\
\text { empresarial } \\
\text { - Conformación de una empresa que } \\
\text { opere el Parque. }\end{array}$ & $\begin{array}{l}\text { - Centro de desarrollo } \\
\text { tecnológico y empresarial } \\
\text { - Subsectorización del Parque } \\
\text { - Programa de Producción más } \\
\text { limpia }\end{array}$ \\
\hline $\begin{array}{c}\text { COSTO DE LA } \\
\text { INVERSIÓN }\end{array}$ & $\$ 4^{\prime} 911.200 .000$ & $\$ 12^{\prime} 641.973 .963$ & $\$ 9.579 .799 .415$ \\
\hline $\begin{array}{c}\text { ESTADO } \\
\text { ACTUAL } \\
\text { (Marzo 2004) }\end{array}$ & $\begin{array}{l}\text { Inaugurado en Octubre de } 2003 \text {. } \\
\text { - Administración propiedad } \\
\text { horizontal. } \\
\text { - } 54 \text { empresarios. } \\
\text { - Operación de: } \\
\text { - Planta de tratamiento de aguas } \\
\text { residuales. } \\
\text {-Centro de Acopio de Residuos. } \\
\text {-Centro tecnológico } \\
\text { (parcialmente). } \\
\text { - Funcionamiento de } \\
\text { restaurantes. }\end{array}$ & $\begin{array}{l}\text { Actualmente hay } 350 \text { empresas de } \\
\text { curtiembres involucradas. } \\
\text { - Implementación de prácticas de } \\
\text { producción más limpia. } \\
\text { - Factibilidad Técnica, económica y } \\
\text { ambiental. } \\
\text { - Diseño integral de planta de } \\
\text { Pelambre y curtición. } \\
\text { - Factibilidad de una planta de } \\
\text { Tratamiento de aguas residuales. } \\
\text { - Comunidad y sector empresarial } \\
\text { informados del proyecto. }\end{array}$ & $\begin{array}{l}\text { Parado. El lote adquirido fue } \\
\text { intervenido por el IDU en } 1997 \text {, } \\
\text { como consecuencia de la } \\
\text { expansión vial en la zona. } \\
\text { - Está definido grupo de } \\
\text { empresarios para su } \\
\text { conformación y se está } \\
\text { haciendo el estudio de } \\
\text { Prefactibilidad. } \\
\text { - Buscando predio para su } \\
\text { ubicación }\end{array}$ \\
\hline PROYECCIONES & $\begin{array}{l}\text { - Continuación de proyectos } \\
\text { ambientales } \\
\text { - Empezar una segunda etapa } \\
\text { para ampliar el parque y asi } \\
\text { ubicar un mayor número } \\
\text { empresarios de Artes gráficas } \\
\text { - Implementación de sistemas de } \\
\text { calidad y de gestión ambiental. } \\
\text { - Implementación del manual de } \\
\text { (BPM) Buenas Prácticas de } \\
\text { manufactura. }\end{array}$ & $\begin{array}{l}\text { - Construcción de un centro de } \\
\text { acopio, centros de especialización, } \\
\text { planta de subproductos, } \\
\text { comercialización y venta, un } \\
\text { laboratorio para análisis de agua y } \\
\text { control para certificación, y prestar } \\
\text { capacitación para empresarios y } \\
\text { empleados. } \\
\text { - Sensibilización en seguridad } \\
\text { industrial y salud ocupacional. } \\
\text { - Mapa del área delimitada }\end{array}$ & $\begin{array}{l}\text { Adquirir un lote siguiendo la } \\
\text { reglamentación sobre el uso del } \\
\text { suelo, para continuar con el } \\
\text { proyecto. }\end{array}$ \\
\hline
\end{tabular}




\begin{tabular}{|c|c|c|c|c|c|}
\hline PROYECTO & \begin{tabular}{|c|} 
MEANDRO DEL \\
SAY \\
$(2000)$ \\
\end{tabular} & $\begin{array}{c}\text { CÁRNICOS DE } \\
\text { GUADALUPE } \\
(2002) \\
\end{array}$ & $\begin{array}{l}\text { PIRT } \\
\text { (2001) }\end{array}$ & $\begin{array}{l}\text { ASOMARMOL } \\
\text { (2001) }\end{array}$ & $\begin{array}{c}\text { PUENTE ARANDA } \\
(\mathbf{2 0 0 0 )}\end{array}$ \\
\hline ORIGEN & $\begin{array}{l}\text { Zona ubicada en la } \\
\text { localidad rá de } \\
\text { Fontibón, dispuesta } \\
\text { por el POT para } \\
\text { crear proyecto } \\
\text { piloto de PIE. }\end{array}$ & $\begin{array}{l}\text { Demanda judicial } \\
\text { por contaminación } \\
\text { del río Tunjuelito. } \\
\text { Convenio de } \\
\text { Producción más } \\
\text { limpia. } \\
\text { Reubicación de } \\
\text { empresarios por } \\
\text { construcción de } \\
\text { obras viales. }\end{array}$ & $\begin{array}{l}\text { Reubicación de } \\
\text { empresarios ubicados en } \\
\text { zonas residenciales de } \\
\text { Bosa y Kennedy. } \\
\text { (Este parque se asemeja al } \\
\text { de Barcelona, en cuanto a } \\
\text { tratamiento de basuras) }\end{array}$ & $\begin{array}{l}\text { Ineficiencia y } \\
\text { poca } \\
\text { compatibilidad } \\
\text { con el } \\
\text { ambiente de } \\
\text { las empresas } \\
\text { ubicadas en el } \\
\text { Barrio Santa } \\
\text { fe. }\end{array}$ & $\begin{array}{l}\text { Zona ubicada en la } \\
\text { localidad de Puente } \\
\text { Aranda } \\
\text { Disposición del POT } \\
\text { para crear proyecto } \\
\text { piloto de PIE. }\end{array}$ \\
\hline OBJETIVOS & $\begin{array}{l}\text { Existencia de } \\
\text { espacios sobre los } \\
\text { cuales se puede } \\
\text { desarrollar un } \\
\text { proceso de } \\
\text { planeación de } \\
\text { localización } \\
\text { industrial. }\end{array}$ & $\begin{array}{l}\text { - Servicios } \\
\text { comunes } \\
\text { - Tratamiento de } \\
\text { agua } \\
\text { - Producción más } \\
\text { limpia. }\end{array}$ & $\begin{array}{l}\text { - } 6 \text { Puntos de acopio con } \\
\text { ubicación equidistante } \\
\text { - } 2 \text { Centros de reciclaje } \\
\text { con zonas especializadas } \\
\text { para carga y descarga, } \\
\text { clasificación y pesaje de } \\
\text { acuerdo a la materia. } \\
\text { - Programa de Producción } \\
\text { más limpia. }\end{array}$ & $\begin{array}{l}\text { - Programa de } \\
\text { producción } \\
\text { más limpia } \\
\text { - Transferencia } \\
\text { tecnológica } \\
\text { - Centro } \\
\text { tecnológico }\end{array}$ & $\begin{array}{l}\text { Desarrollo Industrial } \\
\text { Avanzado con } \\
\text { participación de } \\
\text { diversos sectores } \\
\text { productivos. }\end{array}$ \\
\hline $\begin{array}{c}\text { ESTADO } \\
\text { ACTUAL } \\
\text { (Marzo 2004) }\end{array}$ & $\begin{array}{l}\text { - Estudio de } \\
\text { factibilidad } \\
\text { técnica, ambiental } \\
\text { y económica. } \\
\text { (Identificación de } \\
\text { grupos sinérgicos, } \\
\text { estructuras } \\
\text { básicas y de } \\
\text { administración) } \\
\text { - Delimitación de } \\
\text { la zona para su } \\
\text { conformación } \\
\text { - Grupo de } \\
\text { empresarios sin } \\
\text { definir. }\end{array}$ & $\begin{array}{l}\text { Se han vendido } 125 \\
\text { locales de los } 200 \\
\text { disponibles. } \\
\text { Avances: } \\
\text { - Estudio } \\
\text { Prefactibilidad } \\
\text { - Grupo de } \\
\text { empresarios ya } \\
\text { definido. } \\
\text { - Establecida zona } \\
\text { para la reubicación } \\
\text { - Conformada la } \\
\text { Fiducia para la } \\
\text { compra del lote. }\end{array}$ & $\begin{array}{l}\text { - Reevaluación por } \\
\text { inconvenientes con } \\
\text { puntos de Acopio. } \\
\text { - Estudio Prefactibilidad. } \\
\text { - Por definirse: grupo de } \\
\text { empresarios para su } \\
\text { conformación y sitio de } \\
\text { ubicación. } \\
\text { Costo de la inversión: } \\
\$ 5.700 .000 .000, \text { la } \\
\text { construcción de los } 2 \\
\text { primeros centros de } \\
\text { acopio. }\end{array}$ & $\begin{array}{l}\text { Suspendido. } \\
\text { El resultado } \\
\text { del estudio no } \\
\text { justificó la } \\
\text { construcción } \\
\text { del Parque. }\end{array}$ & $\begin{array}{l}\text { Estudio de } \\
\text { factibilidad téenica, } \\
\text { ambiental y } \\
\text { económica. } \\
\text { Avances: } \\
\text { Interrelaciones } \\
\text { entre las empresas. } \\
\text { - Apoyo del CAM } \\
\text { - Sistema de } \\
\text { adopción o padrinaje } \\
\text { de las compañías } \\
\text { grandes hacia } \\
\text { pequeñas. }\end{array}$ \\
\hline PROYECCIONES & $\begin{array}{l}\text { De acuerdo a } \\
\text { resultados de } \\
\text { estudio de } \\
\text { factibilidad. }\end{array}$ & $\begin{array}{l}\text { Continuar con el } \\
\text { programa de } \\
\text { producción más } \\
\text { limpia, animar a } \\
\text { los empresarios a } \\
\text { que se incorporen } \\
\text { al proyecto. }\end{array}$ & $\begin{array}{l}\text { Inicio de concientización } \\
\text { ciudadana sobre manejo } \\
\text { de residuos domésticos, } \\
\text { mientras se define } \\
\text { ubicación de centros de } \\
\text { acopio. }\end{array}$ & & $\begin{array}{l}\text { De acuerdo a } \\
\text { resultados de estudio } \\
\text { de factibilidad. } \\
\text { Necesidad de } \\
\text { reglamentación de la } \\
\text { U.P.Z y necesidad de } \\
\text { gestión a nivel } \\
\text { gerencial. }\end{array}$ \\
\hline
\end{tabular}

